\title{
Activator protein-1 is a novel regulator of mesencephalic astrocyte-derived neurotrophic factor transcription
}

\author{
CHANG-HUI WANG ${ }^{1-3}$, TONG-CUI JIANG ${ }^{2,4}$, WEI-MIN QIANG ${ }^{2,4}$, LI ZHANG ${ }^{2,4}$, \\ LI-JIE FENG ${ }^{2,4}$, YU-JUN SHEN ${ }^{2,4}$ and YU-XIAN SHEN ${ }^{2,4}$
}

\begin{abstract}
${ }^{1}$ Department of Pharmacology, School of Pharmacy; ${ }^{2}$ Department of Pharmacology, School of Basic Medical Sciences, Anhui Medical University, Hefei, Anhui 230032; ${ }^{3}$ Department of Cardiology, First Affiliated Hospital of Anhui Medical University, Hefei, Anhui 230022; ${ }^{4}$ Biopharmaceutical Research Institute, Anhui Medical University, Hefei, Anhui 230032, P.R. China
\end{abstract}

Received February 14, 2018; Accepted October 3, 2018

DOI: $10.3892 / \mathrm{mmr} .2018 .9601$

\begin{abstract}
Mesencephalic astrocyte-derived neurotrophic factor (MANF) is an endoplasmic reticulum stress-inducible protein, which has been suggested to be upregulated in inflammatory diseases; however, how inflammation regulates its transcription remains unclear. Activator protein-1 (AP-1), which is a transcription factor complex composed of c-Fos and c-Jun, is activated during the inflammatory process. The present study aimed to investigate whether the AP-1 complex regulates MANF transcription. The results of a luciferase reporter assay revealed that one of three putative AP-1 binding sites in the MANF promoter region is essential for enhancement of MANF transcription. Mechanistically, AP-1 was revealed to directly bind to the promoter region of the MANF gene by chromatin immunoprecipitation assay. Furthermore, MANF was strongly expressed in the liver tissues of patients with hepatitis B virus (HBV) infection, compared with in normal liver tissues from patients with hepatic hemangioma. Furthermore, c-Fos and c-Jun were also upregulated in the nuclei of hepatocytes from patients with HBV infection. In mice treated with carbon tetrachloride, the expression patterns of MANF, c-Fos and c-Jun were similar to those in patients with HBV. These results suggested that the AP-1 complex may be a novel regulator of MANF transcription, which may be involved in liver inflammation and fibrosis.
\end{abstract}

\section{Introduction}

Mesencephalic astrocyte-derived neurotrophic factor (MANF) is a recently discovered neurotrophic factor, which was initially isolated from astrocyte culture medium and was

Correspondence to: Professor Yu-Xian Shen, Department of Pharmacology, School of Basic Medical Sciences, Anhui Medical University, 81 Meishan Road, Hefei, Anhui 230032, P.R. China E-mail: shenyx@ahmu.edu.cn

Key words: mesencephalic astrocyte-derived neurotrophic factor, activator protein-1, c-Fos, c-Jun, inflammation, liver fibrosis revealed to selectively exert protective effects on dopaminergic neurons $(1,2)$. Subsequent studies indicated that MANF is induced and secreted in response to experimental endoplasmic reticulum (ER) stress in vitro and in vivo (3-6). MANF has a protective role under physiological and pathophysiological conditions, including in neurodegenerative diseases, ischemic heart disease, diabetes mellitus, chondrodysplasia and polycystic ovary syndrome (4,6-15). Further studies have suggested that MANF protects against various forms of ER stress-induced cell damage via its C-terminal domain. Our previous studies also confirmed that MANF protects neurons in rats with middle cerebral artery occlusion-induced focal cerebral ischemia via alleviating ER stress $(5,7,16,17)$. A recent study identified MANF as an immune modulator that serves a critical role in mediating tissue repair in the retina (18). Our previous study also indicated that MANF is involved in regulation of inflammation. Notably, it is upregulated in patients with rheumatoid arthritis and systemic lupus erythematosus, and in rabbits with antigen-induced arthritis (19). Further studies have demonstrated that MANF is a novel negative regulator of inflammation, functioning as an inhibitor of the nuclear factor $(\mathrm{NF})-\kappa \mathrm{B}$ signaling pathway by blocking the binding of p65 to the promoter of its target genes $(20,21)$. In addition, it has been reported that MANF inhibits oxygen-glucose deprivation-induced cell damage and inflammatory cytokine secretion via suppressing ER stress in rat astrocytes in primary culture (22). Furthermore, MANF may decrease lipopolysaccharide (LPS)-induced proinflammatory cytokines, interleukin (IL)-1 $\beta$, tumor necrosis factor (TNF)- $\alpha$ and interferon- $\gamma$, through regulating the $\mathrm{NF}-\kappa \mathrm{B}$ signaling pathway and phosphorylation of p38-mitogen-activated protein kinases in neural stem cells (23). However, how inflammation regulates MANF expression in inflammatory diseases remains unknown.

Activator protein-1 (AP-1) is a transcription factor complex composed of heterodimers or homodimers of members of the Fos, Jun, activating transcription factor and MAF protein families (24). The c-Fos/c-Jun heterodimer is the prototypic form of the AP-1 complex, which binds to its target DNA motifs to transcriptionally activate target genes (24,25). AP-1 and $\mathrm{NF}-\kappa \mathrm{B}$ are the principal components of two critical inflammatory signaling pathways involved in the response 
to inflammatory stimuli $(26,27)$. Proinflammatory factors, such as TNF- $\alpha$ and IL-1, can activate the AP-1 signaling pathway $(28,29)$. Using the Database of Transcriptional Start Sites (DBTSS) and Transcription Element Search Software (TESS), three putative binding sites of AP-1 were detected in the 5'-flanking region $(-1,239$ to $+176 \mathrm{bp})$ of the human MANF gene in the present study.

Since the effects of AP-1 on MANF expression in inflammatory diseases remain to be elucidated, it was hypothesized that MANF may be a potential target gene of transcriptional activation by AP-1. The present study investigated the association between AP-1 and MANF expression in human liver tissues and a mouse liver injury model. In addition, the binding of AP-1 to the MANF promoter region was analyzed using chromatin immunoprecipitation (ChIP) assays.

\section{Materials and methods}

Collection of human liver tissues. Human liver tissues were obtained from 10 patients with hepatocellular carcinoma (HCC) and HBV infection and four control patients with hepatic hemangioma (HHG); these patients were admitted to the First Affiliated Hospital of Anhui Medical University (Hefei, China) and the Chinese People's Liberation Army 123 Hospital (Bengbu, China) between January and December 2014. General patient information is presented in Table I. All patients received partial hepatectomy. Final diagnosis of the hepatic nodules was confirmed by histological examination of biopsy specimens. All tissue samples were fixed in $4 \%$ neutral buffered formaldehyde at $4^{\circ} \mathrm{C}$ for $24 \mathrm{~h}$ and were embedded in paraffin. The use of human liver tissues was in accordance with the ethical standards of the Declaration of Helsinki. Written informed consent was obtained from all patients and the present study was approved by the Human Research Ethics Committee of Anhui Medical University (license number: 20131359).

Preparation of a murine model of liver fibrosis. A total of 12 C57BL/6J mice (age, 6 weeks; weight, $20 \mathrm{~g}$ ) were obtained from Beijing Vital River Laboratory Animal Technology Co., Ltd. (Beijing, China) and were individually housed in ventilated cages at the Anhui Medical University animal facility. The mice were kept in a controlled temperature $\left(21 \pm 1^{\circ} \mathrm{C}\right)$ and humidity $(50 \pm 5 \%)$ environment, under a $12 \mathrm{~h} \mathrm{light/dark}$ cycle, with ad libitum access to food and water. To induce liver fibrosis, six male mice received an intraperitoneal injection of $2 \mu \mathrm{l} / \mathrm{g}$ carbon tetrachloride $\left(\mathrm{CCl}_{4}\right)$ in sterile olive oil three times per week for 4 weeks. The six control mice were injected with same volume of sterile olive oil three times per week for 4 weeks. The body weight of the mice was monitored each week. The mice were sacrificed after 4 weeks, and livers were removed and were immediately fixed in $4 \%$ paraformaldehyde at $4^{\circ} \mathrm{C}$ for $24 \mathrm{~h}$. All mouse studies were conducted according to protocols approved by the Animal Ethics Committee of Anhui Medical University (license number: 20160329).

Histological analysis of liver fibrosis. Mouse liver sections were stained with hematoxylin at $25^{\circ} \mathrm{C}$ for $8 \mathrm{~min}$ and eosin at $25^{\circ} \mathrm{C}$ for $3 \mathrm{sec}(\mathrm{H} \& \mathrm{E})$, and Sirius red at $25^{\circ} \mathrm{C}$ for $1 \mathrm{~h}(\mathrm{SR})$, in order to evaluate the degree of inflammation and collagen deposition, respectively. The degree of mouse hepatic fibrosis was determined by Masson trichrome staining according to the manufacturer's protocol (cat. no. MST-8003; Fuzhou Maixin Biotechnology Development Co., Ltd., Fuzhou, Fujian, China).

Immunohistochemistry (IHC). Paraffin-embedded mouse and human tissues sections (3-5 $\mu \mathrm{m})$ were deparaffinized and rehydrated prior to IHC for the detection of MANF, c-Fos and c-Jun proteins, as previously described (30). Briefly, antigen retrieval was performed by pressure cooking slides for $5 \mathrm{~min}$ in $0.01 \mathrm{M}$ citrate buffer. Slides were incubated for $30 \mathrm{~min}$ at $37^{\circ} \mathrm{C}$ in $20 \%$ (vol/vol) hydrogen peroxide to block endogenous peroxidase activity and then washed in phosphate-buffered saline (PBS). Following blocking with $10 \%$ normal goat serum, the sections were incubated with primary antibodies, including rabbit anti-MANF (dilution 1:1,000; cat. no. ab126321; Abcam, Cambridge, MA, USA), rabbit anti-c-Fos (dilution 1:100; cat. no. 2250; Cell Signaling Technology, Inc., Danvers, MA, USA) and rabbit anti-c-Jun (dilution 1:250; cat. no. ab32137; Abcam), at $4^{\circ} \mathrm{C}$ overnight, followed by horseradish peroxidase-conjugated secondary antibody at $37^{\circ} \mathrm{C}$ (dilution 1:2,000; cat. no. TA140003, OriGene Technologies, Inc., Beijing, China) for $30 \mathrm{~min}$. Normal IgG (dilution 1:100; cat. no. 3900; Cell Signaling Technology, Inc.) was used as a control. DAB was used for visualization and dark-brown staining was considered positive. Immunohistochemical staining was visualized and images were captured using an Olympus BX35 microscope (Olympus Corporation, Tokyo, Japan); staining was analyzed by a blinded independent pathologist. For analysis, the total cells were counted in $>5$ views from one slide and the positive intensity was measured by ImageJ (version 1.8.0, National Institutes of Health, Bethesda, MD, USA). Briefly, positive staining for each antibody was determined based on the percentage of positive cells ( 0 , negative; $1+$, weak; $2+$, moderate; $3+$, strong). Representative images were collected using the Olympus BX35 microscope.

Cell culture and transfection. The HepG2 human liver cancer cell line and 293 cells, selected for ease of transfection, were obtained from CHI Scientific, Ltd. (Jiangyin, Jiangsu, China). All cells were maintained in Dulbecco's modified Eagle's medium (Gibco; Thermo Fisher Scientific, Inc., Waltham, MA, USA) supplemented with $10 \%$ fetal bovine serum (Tianjin Ankangyuan Technology Development Co., Ltd., Tianjin, China) at $37^{\circ} \mathrm{C}$ in a humidified atmosphere containing $5 \% \mathrm{CO}_{2}$. The HepG2 human liver cancer cells $\left(0.8 \times 10^{5}\right.$ cells per well in 24 -well plates in serum-free medium) were transiently transfected with the pGL3-hMANF plasmids and pcDNA3.1/c-Fos or pcDNA3.1/c-Jun plasmids with $800 \mathrm{ng} / \mathrm{ml}$ at $37^{\circ} \mathrm{C}$ for $24 \mathrm{~h}$ using Lipofectamine ${ }^{\circledR} 2000$ (Invitrogen; Thermo Fisher Scientific, Inc.), according to the manufacturer's protocol.

Prediction of transcription factor binding sites. The putative binding sites for AP-1 in the 5'-flanking region (-1,239 to $+176 \mathrm{bp})$ of the human MANF gene were predicted using the DBTSS (http://dbtss.hgc.jp/index.html, Human Genome Center, Institute of Medical Science, The University of Tokyo, Tokyo, 
Table I. Clinical characteristics of the selected subjects.

\begin{tabular}{lcc}
\hline Characteristic & HBV $(\mathrm{n}=10)$ & HHG $(\mathrm{n}=4)$ \\
\hline Sex, n (\%) & $9(90)$ & $3(75)$ \\
$\quad$ Male & $1(10)$ & $1(25)$ \\
Female & $51.2 \pm 3.9$ & $53.0 \pm 6.7$ \\
Age, years (means \pm standard & & \\
deviation) & & - \\
Etiology, n (\%) & $10(100 \%)$ & - \\
HBV & 0 & - \\
HCV & 0 & \\
Others & & \\
Liver injury, U/l & & \\
(means \pm standard deviation) & $74.2 \pm 69.2$ & $13.8 \pm 4.9$ \\
Serum ALT & $52.8 \pm 42.6$ & $18.5 \pm 1.7$ \\
Serum AST & & \\
\hline
\end{tabular}

ALT, alanine aminotransferase; AST, aspartate aminotransferase; $\mathrm{HBV}$, hepatitis B virus; $\mathrm{HCV}$, hepatitis C virus; HHG, hepatic hemangioma.

Japan) and TESS version 10.0 beta (http://www.cbil.upenn. edu/tess/, Computational Biology and Informatics Laboratory, University of Pennsylvania, Philadelphia, PA, USA).

Cloning and mutagenesis. The 1,415-bp human MANF promoter region spanning $-1,239$ to +176 bp was amplified by polymerase chain reaction (PCR) from human genomic DNA extracted from 293 cells using the primer sets 5'-CCATTG TCCCAAGAGGTATTTT-3' (forward) and 5'-CTATCCCGC ACCTTCGCAG-3' (reverse). PCR products were then ligated into a pMD-18-T vector (cat. no. 6011; Takara Biomedical Technology Co., Ltd, Beijing, China) using KpnI and HindIII, followed by subcloning into the dual luciferase expression vector pGL3-Basic (Promega Corporation, Madison, WI, USA), in order to prepare the recombinant plasmid pGL3-hMANF $(-1,293 /+176)$ expressing the entire promoter region of human MANF. The sequencing results were compared with the human MANF cDNA sequence reported in the GenBank database (https://www.ncbi.nlm.nih.gov/genbank/?). Three truncates, pGL3-hMANF (-880/+83), pGL3-hMANF (-423/+83), and pGL3-hMANF (-265/+83), expressing 963-, 506- and 348-bp fragments of the human MANF promoter region, respectively, were generated using pGL3-hMANF $(-1,293 /+176)$ as a template and the following primer sets: pGL3-hMANF (-880/+83), forward 5'-CGGGGTACCCAGTGCTTCTCTGGTGAT TCCC-3', reverse 5'-GGGAAGCTTCATCCTCCTCATCCT CCTCATC-3'; pGL3-hMANF (-423/+83), forward 5'-CGG GGTACCGTCTTGGCTGACCCCAGAACTC-3', reverse 5'-GGGAAGCTTCATCCTCCTCATCCTCCTCATC-3'; and pGL3-hMANF (-265/+83), forward 5'-CGGGGTACCCCA CACCGCTTCCGTCG-3' and reverse 5'-GGGAAGCTTCAT ССТССТСАТССТССТСАТС-3'. The deletion mutants were subcloned into the luciferase expression vector pGL3-Basic to generate pGL3-hMANF (-880/+83), pGL3-hMANF (-423/+83) and pGL3-hMANF (-265/+83) plasmids.
Mutagenesis of putative AP-1 binding sites located at position -421 and -326 of the MANF promoter was performed using a Multipoints Mutagenesis kit (Takara Bio, Inc., Otsu, Japan), according to the manufacturer's protocol. All mutations/substitutions in the DNA were confirmed by Sanger sequencing (Sangon Biotech Co., Ltd., Shanghai, China).

Luciferase assay. A luciferase assay was performed using a DualLuciferase Reporter Assay system (Promega Corporation), according to the manufacturer's protocol. Briefly, HepG2 cells were seeded in 24 -well plates at a density of $0.8 \times 10^{5}$ cells/well and were cotransfected with the pGL3-hMANF plasmids and c-Fos or c-Jun-expressing plasmids with $800 \mathrm{ng} / \mathrm{ml}$ at $37^{\circ} \mathrm{C}$ for $24 \mathrm{~h}$. The Renilla luciferase reporter plasmid pRL-TK (Promega Corporation) was used as an internal control. Cells were lysed $24 \mathrm{~h}$ post-transfection using lysis buffer (Promega Corporation) and luciferase activity was immediately determined using a GloMax ${ }^{\mathrm{TM}}$ 20/20 luminometer (Promega Corporation). The results were normalized to corresponding pRL-TK activity. Independent experiments were performed at least three times.

ChIP assay. ChIP was performed using a Agarose ChIP assay kit (cat. no. 26156; Thermo Fisher Scientific, Inc.), according to the manufacturer's protocol. The nuclei were isolated from HepG2 liver cancer cells following treatment with phorbol-12-myristate-13-acetate $(10 \mathrm{nM})$ at $37^{\circ} \mathrm{C}$ for $2 \mathrm{~h}$. Chromatin complexes were immunoprecipitated with $4 \mu \mathrm{g}$ anti-c-Jun (cat. no. ab32137; Abcam, Cambridge, MA, USA) or anti-c-Fos (cat. no. ab7963; Abcam), according to the manufacturer's protocol. A parallel immunoprecipitation was carried out using $2 \mu \mathrm{l}$ normal rabbit $\mathrm{IgG}$ in $500 \mu \mathrm{l}$ diluted lysate (cat. no. 26156, Thermo Fisher Scientific, Inc.) as a negative control and $10 \mu \mathrm{l}$ anti-RNA polymerase II antibody in $500 \mu$ l diluted lysate (cat. no. 26156, Thermo Fisher Scientific, Inc.) as a positive control. PCR was performed to amplify a 236-bp fragment containing the putative AP-1 binding sites using appropriate primers (forward, TCACATTCTCACCAG CCACT; reverse, CAGGTCGATCTGCTTGTCATAC), as previously described (21). PCR products were resolved on a $1.5 \%$ agarose gel and were visualized by ethidium bromide (cat. no. 123945-8, Sangon Biotech Co., Ltd.) staining.

Statistical analysis. Data are presented as the means \pm standard deviation. All statistical analyses were performed using GraphPad Prism (GraphPad Software, Inc., La Jolla, CA, USA). Two-way analysis of variance with Tukey's post hoc correction was conducted for multiple comparisons. $\mathrm{P}<0.05$ was considered to indicate a statistically significant difference.

\section{Results}

Screening of putative AP-1 binding sites in the MANF promoter region. To screen the transcriptional regulators of MANF, the promoter region of the human MANF gene within a sequence located 1,239 bp upstream of the transcription start site was analyzed using DBTSS and TESS. Three putative AP-1 binding sites were identified as follows: At -554 bp (5'-GTCA-3'; AP-1-1), at -421 bp (5'-TGAC-3'; AP-1-2) and at -326 bp (5'-TGCA-3'; AP-1-3) (Fig. 1A). 
A

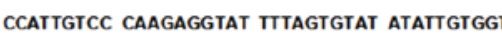

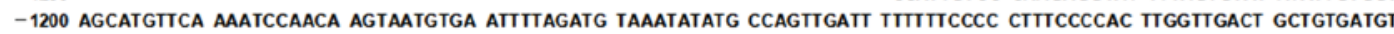
-1100 GAATTAAAGA IAAATACGTG ATACTGATCC AGTGAATICA CTGAATGGGA GCAGAGGAIG TGCCCCTCTC CAACCCTIGT CGCCGGGCTC CAAGCCCTII - 1000 TTAGCCCTAT AGACATGTCC TITTIGCACT GAGTAGAGCC AACGGTTCCC GCATCCTGCC TIGAAGGCAG GCAGCCAGGA GGGCACTTGA AAGGAGGGCA -900 GTCGAAAAGG AAACATCCTC CAGTGCTTCT CTGGTGATTC CCGGGACCCC ACTCAGGAGG TCCCACGGCA GCGGGGGACC CCGAGGGCCT AGCCGGGGAG -800 GCAGGAGGAG GCTGGCCAGC CACCAAGCCA CGATTCAGAG CTTGTAAGGG CACCTCCCGA GCCCCTCACC TTCCCACTCT GAGGCGACCC CATGTCCTCG -700 ACCGATGATC TGGCCCGGGC GCTTCCCTCC ACGGTCTAGA GTGGCGGCCC AGCTITGAGC TTGGCGCGCC TTGAGCCCTT CCTTCAGATG TCCCGCAGTC -600 CGTCCACCCC GCACGTCTCC TATCAGCCTA TGCACGGCGG GCTCCGGTCA GGTGGAGGAT CAGCAGGTGG GCTITCAAAG GTTGGATCCG ITGCCCACGC AP-1-1

-500 GAATGAAAGA AGAAAAAAGA AACCTCTTTG AGCCTTGCCT GCGGCCGCCC GCCCTTCCTG CCTAACCTGT TGTCTTGGCT GACCCCAGAA CTCCTGCACC AP-1-2

- 400 ACGAGTCGGA GTCCGGGCCA GGAGATCAGC AGGGGTTITC GAGGGAGCCT GGGGCCCAGG GCAGGGGTAC GCGGGICAAC TCAACAGATG TAAGGCGTGG $\overline{\mathrm{AP}-1-3}$

- 300 CCGAACCCCA TICAGCTAGC AGIACCCAGC CTCAGCCACA GICGCTGCCC TICCCAAGA TGGCGGCCCA CACCGCTIGC GTCGCTGCTG TAGTCGCITC - 200 CTGCGGCCAG CCCGGGITCA ATCAGCGGCC GACAACTGTC TAGGGCTCAG ACACCACCAG CCAATGAGGG AGGGCACCGT CCACCCCCCC GTCTGGGCTC

- 100 GCGGCTCCTG GACCAATGGG GAAGTGGCAT GTGGGAGGGC GCCGGGGGGC CCCCCGCCAA TGGGGAGCTA CGGCGCGCGG CCGGGACTTG GCGGCGGTGC $+1 \underset{\text { GGCGCGGCGG GTGCGGTTCA GTCGGTCGGG GGCGGCAGCG GACGACGAGG AGGAGGAGGA GGATGACGAG GATGAGGAGG ATGTGGGCCA CGCAGGGGCT }}{\longrightarrow}$ +101 GGCGGTGGCG CTGGCTCTGA GCGTGCTGCC GGGCAGCCGG GCGCTGCGGC CGGGCGACTG CGAAGGTGCG GGATAG

B MANF promoter

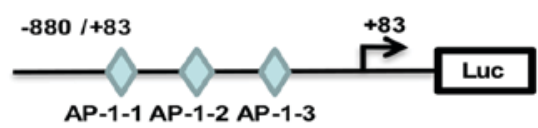

PGL3-hMANF $(-880 /+83)$

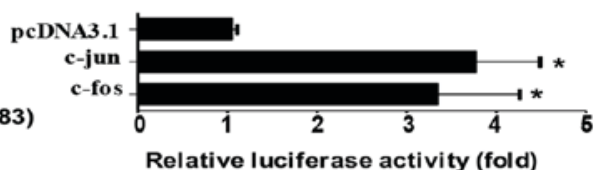

C

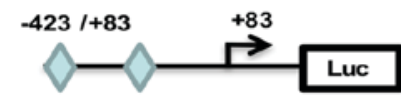

AP-1-2 AP-1-3
pGL3-hMANF (-423/+83) $\quad \begin{gathered}\text { c-jun } \\ \text { c-fos }\end{gathered}$

Relative luciferase activity (fold)

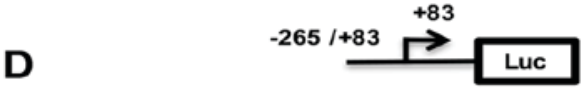

Luc
PGL3-hMANF $(-265 /+83)$

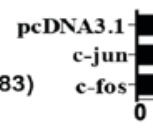

E

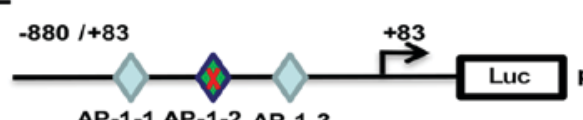

AP-1-1 AP-1-2 AP-1-3

$\mathbf{F}$

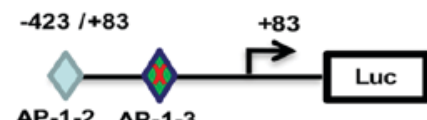

AP-1-2 AP-1-3
PGL3-hMANF $(-880 /+83)$

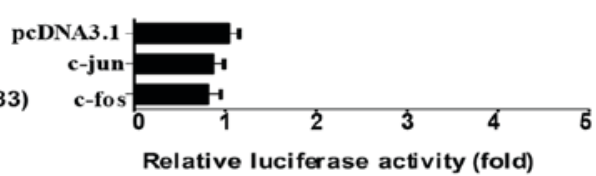

pcD

PGL3-hMANF $(-423 /+83)$
Relative luciferase activity (fold)

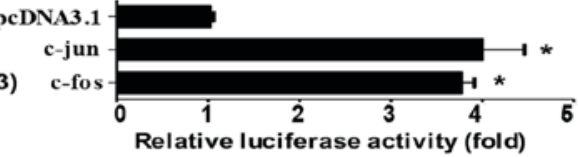

G

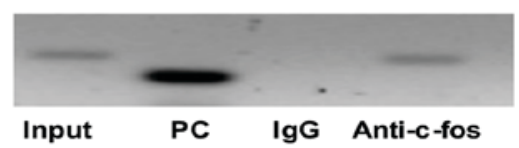

H

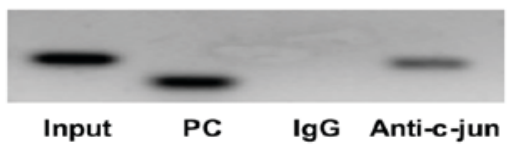

Figure 1. AP-1 binds to the MANF promoter region and regulates MANF transcription. (A) Prediction of three putative AP-1 binding sites in the promoter region of the human MANF gene. (B-D) Overexpression of c-Fos or c-Jun enhanced MANF transcription. Luciferase assay was performed in HepG2 liver cancer cells transfected with plasmids expressing c-Fos or c-Jun together with (B) MANF promoter fragment (-880/+83) containing all three putative AP-1 binding sites, (C) truncate $(-423 /+83)$ containing two of the putative AP-1 binding sites, or (D) truncate $(-265 /+83)$ without the putative AP-1 binding sites cloned in pGL3 basic. (E and F) Effects of mutation of the putative AP-1 binding sites on MANF transcriptional activity. (E) Mutation of AP-1-2 abolished the enhancement of MANF transcription by c-Fos or c-Jun. (F) AP-1-3 mutation had no effect on the enhancement of MANF transcription by c-Fos or c-Jun. (G and H) Binding of c-Fos or c-Jun to the MANF promoter region. The nuclei were isolated from HepG2 liver cancer cells following treatment with phorbol-12-myristate-13-acetate $(10 \mathrm{nM})$ for $2 \mathrm{~h}$, and a ChIP assay was performed with antibodies against (G) c-Fos or (H) c-Jun. Normal goat IgG and anti-RNA polymerase II antibody were used as a negative control and PC, respectively. Data are presented as the means \pm standard deviation. The experiments were repeated at least three times. ${ }^{*} \mathrm{P}<0.05$, compared with the pcDNA3.1 empty vector. AP-1, activator protein -1 ; IgG, immunoglobulin G; MANF, mesencephalic astrocyte-derived neurotrophic factor; PC, positive control.

Identification of the AP-1 binding site involved in MANF transcriptional regulation. To confirm the aforementioned sites as AP-1 binding sites, the MANF promoter region and the truncates were cloned into the pGL3-Basic vector to form pGL3-hMANF (-880/+83) (Fig. 1B), pGL3-hMANF
(-423/+83) (Fig. 1C) and pGL3-hMANF (-256/+83) (Fig. 1D). Subsequently, luciferase activity was detected after co-transfecting HepG2 liver cells with the pGL3-hMANF plasmids and c-Fos or c-Jun-expressing plasmids. The plasmids were successfully transfected, as verified using 

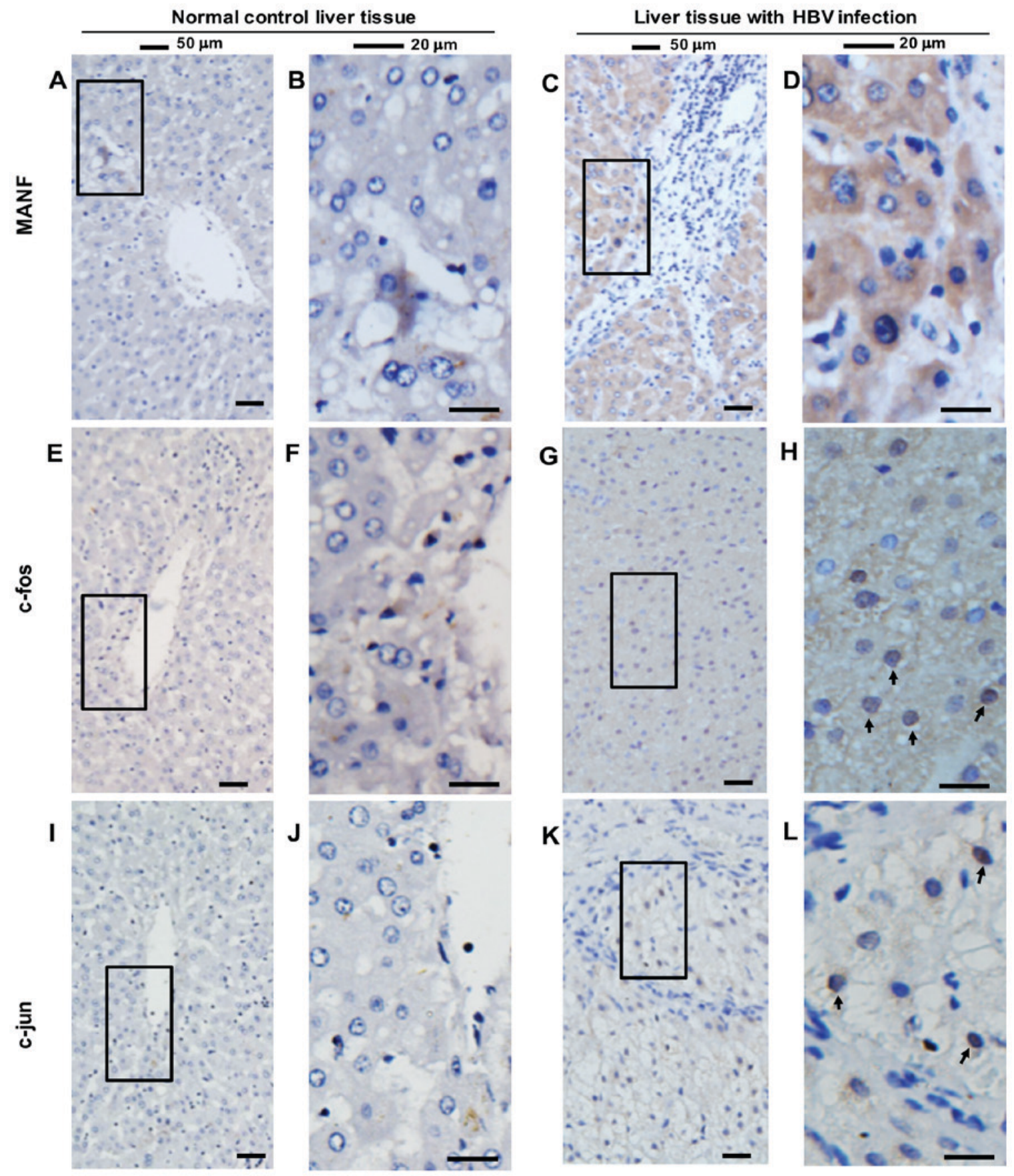

Figure 2. Expression of MANF and AP-1 complex in the liver tissues of patients with HBV infection. (A-D) MANF expression was detected in liver tissues from patients with (A and B) HHG and (C and D) HBV. (B) and (D) are higher magnification images of the areas shown in (A) and (C), respectively. (E-H) c-Fos expression was detected in liver tissue from patients with (E and F) HHG and $(\mathrm{G}$ and $\mathrm{H}) \mathrm{HBV}$. (F) and $(\mathrm{H})$ are higher magnification images of the areas shown in (E) and (G), respectively. (I-L) c-Jun expression was detected in liver tissues from patients with (I and J) HHG and (K and L) HBV. (I) and (K) are higher magnification images of the areas shown in $(\mathrm{J})$ and $(\mathrm{L})$, respectively. Arrows indicate positive cells. HBV, hepatitis B virus; HHG, hepatic hemangioma; MANF, mesencephalic astrocyte-derived neurotrophic factor.

immunofluorescence and western blotting (data not shown). As shown in Fig. 1B-D, c-Jun and c-Fos were able to significantly increase the luciferase activity of cells transfected with plasmids containing the MANF promoter and the $-423 /+83$ truncate, but not the $-256 /+83$ truncate, which does not contain the putative AP-1 binding site. In addition, there was no significant difference in luciferase activity between pGL3-hMANF (-880/+83) and pGL3-hMANF (-423/+83) truncates was observed (Fig. 1B and C), thus suggesting that the AP-1-1 binding site may not be required for regulating
MANF transcription. These findings indicated that the two putative binding sites AP-1-2 and AP-1-3 are required for the enhancement of MANF promoter activity by AP-1.

To further confirm the essential binding sites for AP-1 in the MANF promoter region, mutations were introduced into the putative binding sites AP-1-2 (TGAC $\rightarrow$ CAGC) (Fig. 1E) and AP-1-3 (GTCA $\rightarrow$ CAGC) (Fig. 1F). HepG2 liver cells were co-transfected with the mutated plasmids and c-Fos or c-Jun, and the reporter activity was detected. The results revealed that the AP-1-2 mutation abolished the enhancement of MANF 

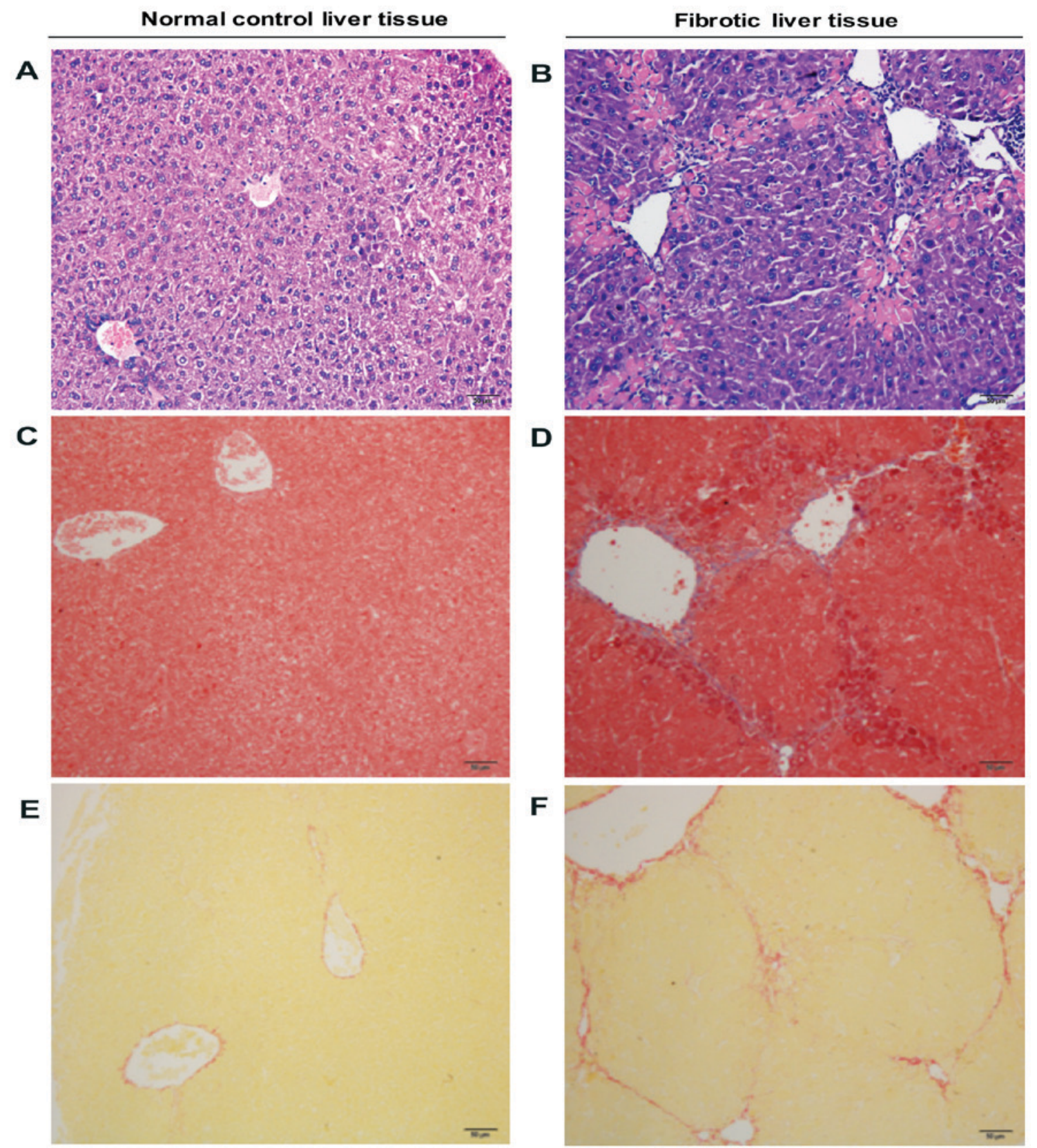

Figure 3. Histological analysis of liver fibrosis in mice. (A and B) Hematoxylin and eosin staining; (C and D) Masson trichrome staining; (E and F) Sirius red staining. (A, C and E) Tissues from normal control mice; (B, D and F) tissues from model mice treated with carbon tetrachloride. Scale bar, $50 \mu \mathrm{m}$. MANF, mesencephalic astrocyte-derived neurotrophic factor.

promoter activity by c-Fos or c-Jun (Fig. 1E). However, the AP-1-3 mutation did not alter the effects of c-Fos and c-Jun on MANF promoter activity (Fig. 1F), thus suggesting that AP-1-2 (5'-TGAC-3') is the essential binding site of AP-1 required for MANF transcriptional regulation.

AP-1 directly binds to the promoter region of the MANF gene. The present study performed ChIP analysis to validate the direct binding of AP-1 to the MANF promoter region. For this experiment, ChIP with anti-c-Fos and c-Jun antibodies was performed. The pulled down DNA was subjected to PCR (Fig. 1G, lane 4 and Fig. 1H, lane 4) to amplify the MANF promoter region containing the AP-1 binding site. As shown in Fig. $1 \mathrm{G}$ and $\mathrm{H}, \mathrm{c}-\mathrm{Fos}$ and c-Jun were revealed to bind to the MANF promoter, thus suggesting that upregulation of MANF may be caused by the direct binding of AP-1 to the promoter region of the MANF gene.

Expression of MANF and AP-1 in human and murine liver samples. Previous studies have demonstrated that MANF is an ER stress-inducible protein (3), which is upregulated in inflammatory diseases $(19,21)$. To determine the expression of MANF in human liver samples, liver tissues were collected from 10 patients with hepatocellular carcinoma (HCC) and $\mathrm{HBV}$ infection, and from four patients with $\mathrm{HHG}$ (Fig. 2). It was revealed that MANF was strongly expressed in the paracancerous liver tissue of five patients with HCC and HBV infection (Fig. 2C and D), compared with in normal liver tissues from patients with HHG (Fig. 2A and B). Additionally, it was revealed that c-Fos and c-Jun were upregulated in the nuclei 


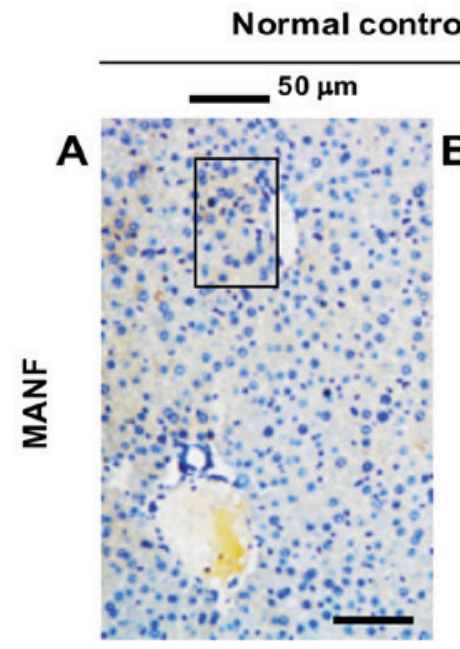

\section{ol liver tissue}
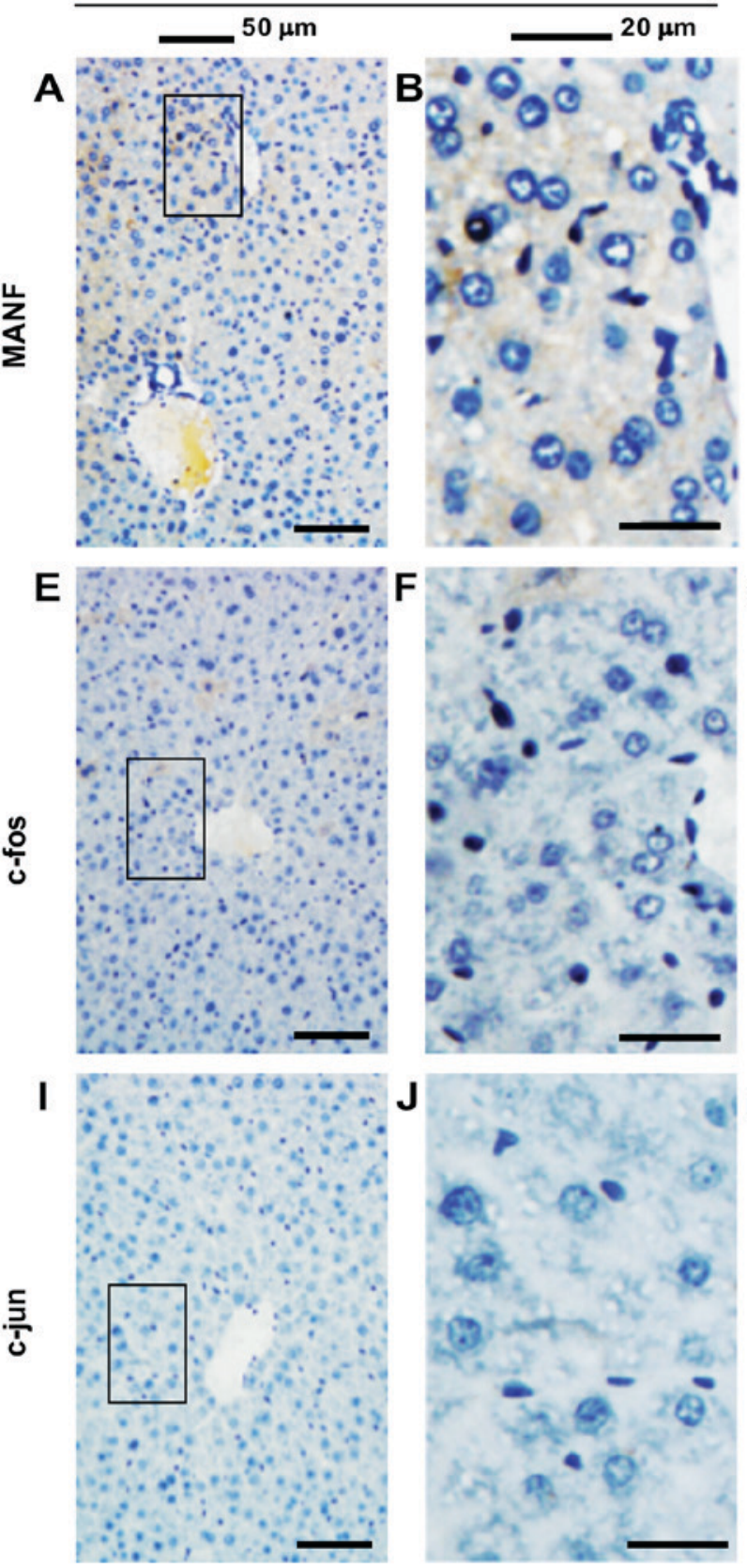

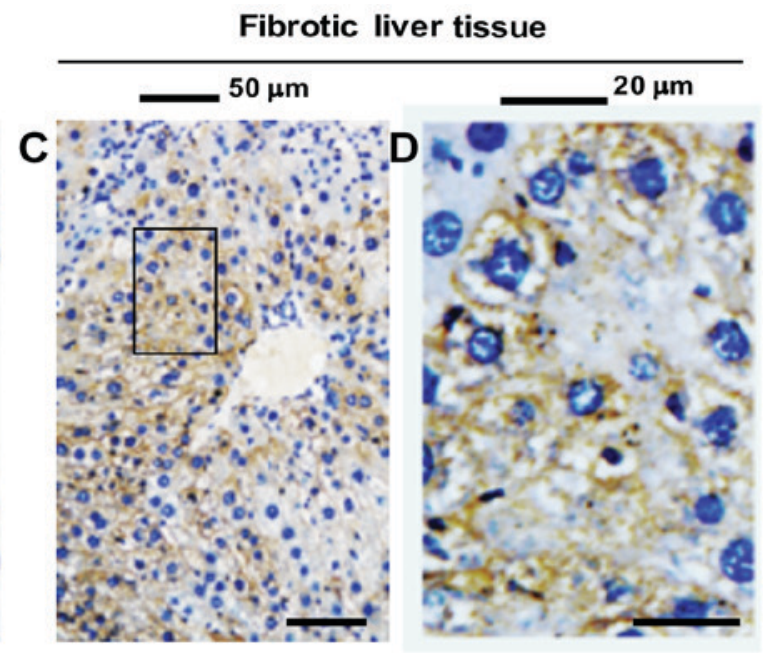

Fibrotic liver tissue
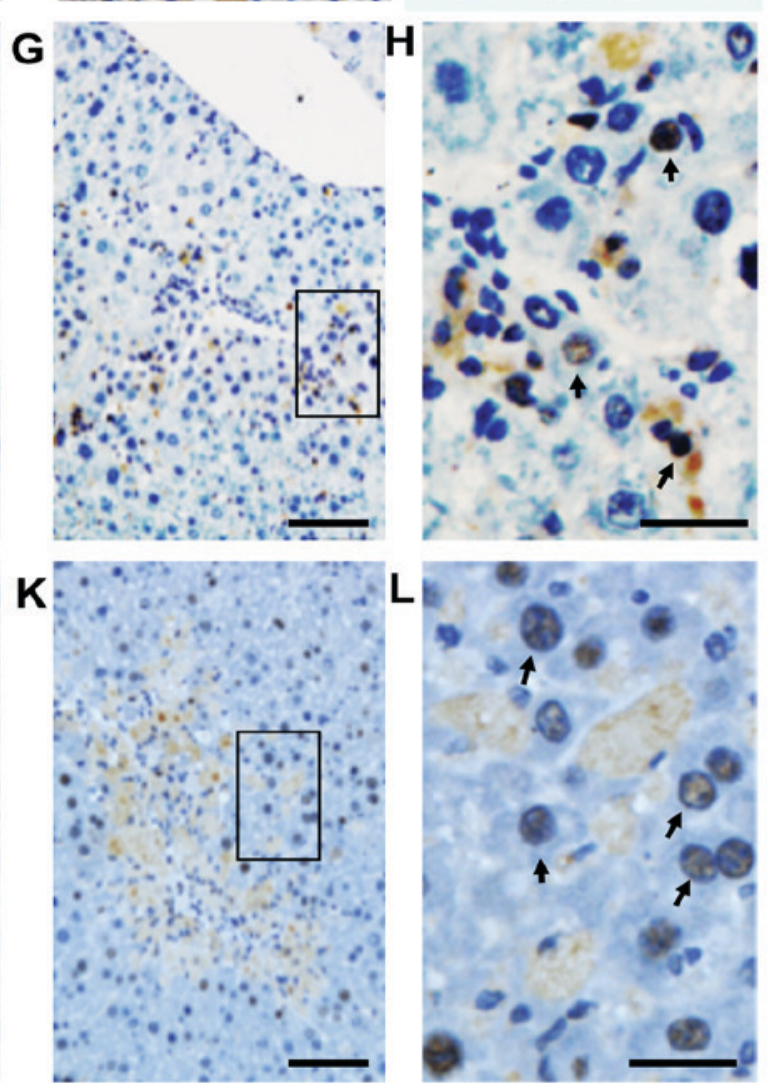

Figure 4. Expression of MANF and AP-1 complex in the liver tissues of mice treated with carbon tetrachloride. (A-D) MANF expression was detected in the (A and B) normal control liver tissues and (C and D) fibrotic liver tissues. (B) and (D) are higher magnification images of the areas shown in (A) and (C), respectively. (E-H) c-Fos expression was detected in (E and F) normal control liver tissues and $(\mathrm{G}$ and $\mathrm{H})$ fibrotic liver tissues. $(\mathrm{F})$ and $(\mathrm{H})$ are higher magnification images of the areas shown in (E) and (G), respectively. (I-L) c-Jun expression was detected in (I and J) normal control liver tissues and (K and L) fibrotic liver tissues. (I) and (K) are higher magnification images of the areas shown in (J) and (L), respectively. Arrows indicate positive cells. MANF, mesencephalic astrocyte-derived neurotrophic factor.

of hepatocytes of patients with HBV infection (Fig. 2E-L, indicated by arrows).

MANF expression was also detected in liver tissues from mice treated with $\mathrm{CCl}_{4}$. In the mouse liver injury model, $\mathrm{CCl}_{4}$-induced liver fibrosis was initially evaluated via $\mathrm{H} \& \mathrm{E}$, $\mathrm{SR}$ and Masson trichrome staining. As shown in Fig. 3, $\mathrm{CCl}_{4}$ treatment for 4 weeks resulted in liver inflammation and fibrosis. Furthermore, it was revealed that few MANF-positive cells were detected in the liver samples obtained from the control group. Conversely, in $\mathrm{CCl}_{4}$-treated mice, the majority of hepatocytes were MANF-positive (Fig. 4A-D). Coincidentally,
c-Fos and c-Jun were also strongly expressed in the nuclei of hepatocytes in $\mathrm{CCl}_{4}$-treated mice, compared with in the control mice (Fig. 4E-L). These results suggested that MANF and AP-1 may be involved in liver inflammation, and that MANF expression may be associated with AP-1 under such a condition.

\section{Discussion}

Our previous studies demonstrated that MANF is upregulated under various conditions, including ischemia and hypoxia, 
and in response to inflammatory stimuli, all of which are associated with ER stress $(5,7,19,21)$. However, how MANF is upregulated in response to ER stress, particularly in an inflammatory state, remains unclear. A previous study demonstrated that the unfolded protein response is able to induce mouse MANF expression via ER stress-responsive element (ERSE) II (31). Recently, it was reported that ERSE I serves a dominant role in mediating X-box binding protein 1-induced MANF expression, whereas ERSE II exerts little impact on MANF transcription (32). The present study demonstrated that the AP-1 complex, comprised of c-Fos and c-Jun subunits, may enhance MANF transcription through binding to the sequence TGAC (-421/-418 bp) within the MANF promoter region. Collectively these findings suggested that MANF transcription may be regulated by numerous mechanisms. The current findings may aid to understand how MANF is regulated under inflammatory conditions.

AP-1 is part of an important inflammatory signaling pathway and is activated by LPS and TNF- $\alpha$ via the signaling pathways of TNF receptor-associated factor 6 and transforming growth factor- $\beta$-activated kinase $1(33,34)$. Activation of the AP-1 signaling pathway promotes the expression of inflammatory factors, including IL-1 $\beta$, IL-6 and inducible nitric oxide synthase (35). The present study revealed that MANF was a downstream target, which was upregulated by c-Fos and c-Jun as part of the AP-1 complex, via a direct binding interaction with the MANF gene promoter. In addition, only one binding site was effective in enhancing MANF transcription among the three putative AP-1 binding sites identified. Unlike other AP-1 targets, MANF reportedly exerts anti-inflammatory activity by negatively regulating the NF- $\kappa \mathrm{B}$ signaling pathway (21), thus suggesting that the AP-1 signaling pathway may be involved in dual-directional regulation of inflammation.

Previous studies have reported that HBV replication and expression of hepatoviral proteins, such as $\mathrm{HBV}$ protein $\mathrm{X}$, are associated with potent activation of AP-1 (36-39), and that AP-1 expression is increased in HBV $(40,41)$. Upregulation of AP-1 has also been reported in mouse liver fibrosis induced by $\mathrm{CCl}_{4}$ treatment and common bile duct ligation $(42,43)$, which is consistent with the present findings. Although c-Fos and c-Jun were initially considered as oncogenes, it has been revealed that c-Fos can suppress the growth of murine hepatocytes by inducing cell cycle inhibition and cell death (44). Regarding the mechanisms underlying the effects of AP-1 activation on liver fibrosis, this likely occurs via the ER stress response/extracellular signal-regulated kinase (ERK)/AP-1 signaling pathway, as it has been reported that ER stress inducers activate AP-1-associated genes, including c-Fos and c-Jun, in an ERK-dependent manner in hepatic cells and murine livers (45). In the present study, MANF expression was increased in the liver tissues of patients with HBV infection and of mice with $\mathrm{CCl}_{4}$-induced liver fibrosis. Therefore, it may be hypothesized that the increase in MANF expression in the liver may partially be stimulated by AP-1. However, the impact of MANF on liver inflammation requires further investigation. We have prepared hepatocyte-specific MANF knockout mice and mono-macrophage-specific MANF knockout mice, and aim to compare liver inflammation and fibrosis in wild-type mice and MANF knockout mice in the future; in addition, the underlying mechanisms will be investigated.

In conclusion, the present study demonstrated that the AP-1 complex may be a novel regulator of MANF transcriptional enhancement, and that MANF is a novel downstream target of AP-1, which may indicate a novel role of AP-1 in regulating inflammatory pathways. Therefore, targeting the AP-1/MANF signaling pathway may be a potential therapeutic strategy for the treatment of inflammation and liver injury.

\section{Acknowledgements}

Not applicable.

\section{Funding}

The present study was supported by the National Natural Science Foundation of China (grant nos. 91129729, 81372576 and 81673438 awarded to YXS) and the Youth Fund Project of the First Affiliated Hospital of Anhui Medical University (grant no. 2009kj19 awarded to CHW).

\section{Availability of data and materials}

The datasets used and/or analyzed during the current study are available from the corresponding author on reasonable request.

\section{Authors' contributions}

CHW performed the experiments and wrote the manuscript; TCJ, WMQ and LZ performed some of the experiments; YJS and LJF helped to analyze the data and revised the manuscript; YXS designed the experiments and wrote the manuscript. All authors read and approved the final manuscript.

\section{Ethics approval and consent to participate}

The use of human liver tissues was in accordance with the ethical standards of the Declaration of Helsinki. Written informed consent was obtained from all patients and the present study was approved by the Human Research Ethics Committee of Anhui Medical University (license number: 20131359). All mouse studies were conducted according to protocols approved by the Animal Ethics Committee of Anhui Medical University (license number: 20160329).

\section{Patient consent for publication}

Not applicable.

\section{Competing interests}

The authors declare that they have competing interests.

\section{References}

1. Petrova P, Raibekas A, Pevsner J, Vigo N, Anafi M, Moore MK, Peaire AE, Shridhar V, Smith DI, Kelly J, et al: MANF: A new mesencephalic, astrocyte-derived neurotrophic factor with selectivity for dopaminergic neurons. J Mol Neurosci 20: 173-188, 2003. 
2. Airavaara M, Shen H, Kuo CC, Peränen J, Saarma M, Hoffer B and Wang Y: Mesencephalic astrocyte-derived neurotrophic factor reduces ischemic brain injury and promotes behavioral recovery in rats. J Comp Neurol 515: 116-124, 2009.

3. Apostolou A, Shen Y, Liang Y, Luo J and Fang S: Armet, a UPR-upregulated protein, inhibits cell proliferation and ER stress-induced cell death. Exp Cell Res 314: 2454-2467, 2008.

4. Tadimalla A, Belmont PJ, Thuerauf DJ, Glassy MS, Martindale JJ, Gude N, Sussman MA and Glembotski CC: Mesencephalic astrocyte-derived neurotrophic factor is an ischemia-inducible secreted endoplasmic reticulum stress response protein in the heart. Circ Res 103: 1249-1258, 2008.

5. Yu YQ, Liu LC, Wang FC, Liang Y, Cha DQ, Zhang JJ, Shen YJ, Wang HP, Fang S and Shen YX: Induction profile of MANF/ARMET by cerebral ischemia and its implication for neuron protection. J Cereb Blood Flow Metab 30: 79-91, 2010.

6. Glembotski CC, Thuerauf DJ, Huang C, Vekich JA, Gottlieb RA and Doroudgar S: Mesencephalic astrocyte-derived neurotrophic factor protects the heart from ischemic damage and is selectively secreted upon sarco/endoplasmic reticulum calcium depletion. J Biol Chem 287: 25893-25904, 2012

7. Yang W, Shen Y, Chen Y, Chen L, Wang L, Wang H, Xu S, Fang S, Fu Y, Yu Y and Shen Y: Mesencephalic astrocyte-derived neurotrophic factor prevents neuron loss via inhibiting ischemia-induced apoptosis. J Neurol Sci 344: 129-138, 2014

8. Stratoulias V and Heino TI: MANF silencing, immunity induction or autophagy trigger an unusual cell type in metamorphosing Drosophila brain. Cell Mol Life Sci 72: 1989-2004, 2015.

9. Stratoulias V and Heino TI: Analysis of the conserved neurotrophic factor MANF in the Drosophila adult brain. Gene Expr Patterns 18: 8-15, 2015.

10. Palgi M, Greco D, Lindström R, Auvinen P and Heino TI: Gene expression analysis of Drosophila Manf mutants reveals perturbations in membrane traffic and major metabolic changes. BMC Genomics 13: 134, 2012.

11. Palgi M, Lindström R, Peränen J, Piepponen TP, Saarma M and Heino TI: Evidence that DmMANF is an invertebrate neurotrophic factor supporting dopaminergic neurons. Proc Natl Acad Sci USA 106: 2429-2434, 2009.

12. Lindholm P, Voutilainen MH, Laurén J, Peränen J, Leppänen VM, Andressoo JO, Lindahl M, Janhunen S, Kalkkinen N, Timmusk T, et al: Novel neurotrophic factor CDNF protects and rescues midbrain dopamine neurons in vivo. Nature 448: 73-77, 2007.

13. Lindahl M, Danilova T,PalmE, Lindholm P, Võikar V,HakonenE, Ustinov J, Andressoo JO, Harvey BK, Otonkoski T, et al: MANF is indispensable for the proliferation and survival of pancreatic $\beta$ cells. Cell Rep 7: 366-375, 2014.

14. Wang N, Wang L, Le F, Zhan Q, Zheng Y, Ding G, Chen X, Sheng J, Dong M, Huang H and Jin F: Altered expression of Armet and Mrlp51 in the oocyte, preimplantation embryo, and brain of mice following oocyte in vitro maturation but postnatal brain development and cognitive function are normal Reproduction 142: 401-408, 2011.

15. Cameron TL, Bell KM, Tatarczuch L, Mackie EJ, Rajpar MH, McDermott BT, Boot-Handford RP and Bateman JF: Transcriptional profiling of chondrodysplasia growth plate cartilage reveals adaptive ER-stress networks that allow survival but disrupt hypertrophy. PLoS One 6: e24600, 2011.

16. Shen YX, Sun AM, Fang S, Feng LJ, Li Q, Hou HL, Liu C, Wang HP, Shen JL, Luo J and Zhou JN: Hrd1 facilitates tau degradation and promotes neuron survival. Curr Mol Med 12: 138-152, 2012

17. Wang XY, Song MM, Bi SX, Shen YJ, Shen YX and Yu YQ: MRI dynamically evaluates the therapeutic effect of recombinant human MANF on ischemia/reperfusion injury in rats. Int J Mol Sci 17: E1476, 2016.

18. Neves J, Zhu J, Sousa-Victor P, Konjikusic M, Riley R, Chew S, Qi Y, Jasper H and Lamba DA: Immune modulation by MANF promotes tissue repair and regenerative success in the retina. Science 353: aaf3646, 2016.

19. Wang J, Cheng Q, Wang X, Zu B, Xu J, Xu Y, Zuo X, Shen Y, Wang $J$ and Shen Y: Deficiency of IRE1 and PERK signal pathways in systemic lupus erythematosus. Am J Med Sci 348: 465-473, 2014

20. Wang H, Ke Z, Alimov A, Xu M, Frank JA, Fang S and Luo J: Spatiotemporal expression of MANF in the developing rat brain. PLoS One 9: e90433, 2014.
21. Chen L, Feng L, Wang X, Du J, Chen Y, Yang W, Zhou C, Cheng L, Shen Y, Fang S, et al: Mesencephalic astrocyte-derived neurotrophic factor is involved in inflammation by negatively regulating the NF-kappaB pathway. Sci Rep 5: 8133, 2015.

22. Zhao H, Liu Y, Cheng L, Liu B, Zhang W, Guo YJ and Nie L: Mesencephalic astrocyte-derived neurotrophic factor inhibits oxygen-glucose deprivation-induced cell damage and inflammation by suppressing endoplasmic reticulum stress in rat primary astrocytes. J Mol Neurosci 51: 671-678, 2013.

23. Zhu W, Li J, Liu Y, Xie K, Wang L and Fang J: Mesencephalic astrocyte-derived neurotrophic factor attenuates inflammatory responses in lipopolysaccharide-induced neural stem cells by regulating NF-kappaB and phosphorylation of p38-MAPKs pathways. Immunopharmacol Immunotoxicol 38: 205-213, 2016.

24. Eferl R and Wagner EF: AP-1: A double-edged sword in tumorigenesis. Nat Rev Cancer 3: 859-868, 2003.

25. Gustems M, Woellmer A, Rothbauer U, Eck SH, Wieland T, Lutter D and Hammerschmidt W: c-Jun/c-Fos heterodimers regulate cellular genes via a newly identified class of methylated DNA sequence motifs. Nucleic Acids Res 42: 3059-3072, 2014.

26. Adcock IM: Transcription factors as activators of gene transcription: AP-1 and NF-kappa B. Monaldi Arch Chest Dis 52: 178-186, 1997.

27. Hsu TC, Young MR, Cmarik J and Colburn NH: Activator protein 1 (AP-1)- and nuclear factor kappaB (NF-kappaB)-dependent transcriptional events in carcinogenesis. Free Radic Biol Med 28: $1338-1348,2000$

28. Brenner DA, O'Hara M, Angel P, Chojkier M and Karin M: Prolonged activation of jun and collagenase genes by tumour necrosis factor-alpha. Nature 337: 661-663, 1989.

29. Yu Y, Ge N, Xie M, Sun W, Burlingame S, Pass AK, Nuchtern JG Zhang D, Fu S, Schneider MD, et al: Phosphorylation of Thr-178 and Thr-184 in the TAK1 T-loop is required for interleukin (IL)-1-mediated optimal NFkappaB and AP-1 activation as well as IL-6 gene expression. J Biol Chem 283: 24497-24505, 2008.

30. Liu J, Sha M, Wang Q, Ma Y, Geng X, Gao Y, Feng L, Shen Y and Shen Y: Small ubiquitin-related modifier $2 / 3$ interacts with $p 65$ and stabilizes it in the cytoplasm in HBV-associated hepatocellular carcinoma. BMC Cancer 15: 675, 2015.

31. Mizobuchi N, Hoseki J, Kubota H, Toyokuni S, Nozaki J, Naitoh M, Koizumi A and Nagata K: ARMET is a soluble ER protein induced by the unfolded protein response via ERSE-II element. Cell Struct Funct 32: 41-50, 2007.

32. Wang D, Hou C, Cao Y, Cheng Q, Zhang L, Li H, Feng L and Shen Y: XBP1 activation enhances MANF expression via binding to endoplasmic reticulum stress response elements within MANF promoter region in hepatitis B. Int J Biochem Cell Biol 99: 140-146, 2018.

33. Mackman N, Brand K and Edgington TS: Lipopolysaccharidemediated transcriptional activation of the human tissue factor gene in THP-1 monocytic cells requires both activator protein 1 and nuclear factor kappa B binding sites. J Exp Med 174: 1517-1526, 1991.

34. Mao R, Fan Y, Mou Y, Zhang H, Fu S and Yang J: TAK1 lysine 158 is required for TGF- $\beta$-induced TRAF6-mediated Smad-independent IKK/NF-kappaB and JNK/AP-1 activation. Cell Signal 23: 222-227, 2011

35. Guha M and Mackman N: LPS induction of gene expression in human monocytes. Cell Signal 13: 85-94, 2001.

36. Arzumanyan A, Reis HM and Feitelson MA: Pathogenic mechanisms in HBV- and $\mathrm{HCV}$-associated hepatocellular carcinoma. Nat Rev Cancer 13: 123-135, 2013.

37. Fuest M, Willim K, MacNelly S, Fellner N, Resch GP, Blum HE and Hasselblatt P: The transcription factor c-Jun protects against sustained hepatic endoplasmic reticulum stress thereby promoting hepatocyte survival. Hepatology 55: 408-418, 2012.

38. Twu JS, Lai MY, Chen DS and Robinson WS: Activation of protooncogene $\mathrm{c}$-jun by the $\mathrm{X}$ protein of hepatitis $\mathrm{B}$ virus. Virology 192: 346-350, 1993.

39. Benn J, Su F, Doria M and Schneider RJ: Hepatitis B virus HBx protein induces transcription factor AP-1 by activation of extracellular signal-regulated and c-Jun N-terminal mitogen-activated protein kinases. J Virol 70: 4978-4985, 1996.

40. Kanda T, Yokosuka O, Nagao K and Saisho H: State of hepatitis $\mathrm{C}$ viral replication enhances activation of $\mathrm{NF}-\kappa \mathrm{B}-$ and AP-1-signaling induced by hepatitis B virus X. Cancer Lett 234: $143-148,2006$. 
41. Guo L, Guo Y, Xiao S and Shi X: Protein kinase p-JNK is correlated with the activation of AP-1 and its associated Jun family proteins in hepatocellular carcinoma. Life Sci 77: 1869-1878, 2005.

42. Chu X, Wang H, Jiang YM, Zhang YY, Bao YF, Zhang X, Zhang JP, Guo H, Yang F, Luan YC and Dong YS: Ameliorative effects of tannic acid on carbon tetrachloride-induced liver fibrosis in vivo and in vitro. J Pharmacol Sci 130: 15-23, 2016.

43. Wang Y, Ma J, Chen L, Xie XL and Jiang H: Inhibition of focal adhesion kinase on hepatic stellate-cell adhesion and migration. Am J Med Sci 353: 41-48, 2017.
44. Mikula M, Gotzmann J, Fischer AN, Wolschek MF, Thallinger C, Schulte-Hermann R, Beug H and Mikulits W: The proto-oncoprotein c-Fos negatively regulates hepatocellular tumorigenesis. Oncogene 22: 6725-6738, 2003.

45. Olivares S, Green RM and Henkel AS: Endoplasmic reticulum stress activates the hepatic activator protein 1 complex via mitogen activated protein kinase-dependent signaling pathways. PLoS One 9: e103828, 2014. 